\title{
Spectroscopy with dynamical Chirally Improved quarks
}

\author{
Christof Gattringer ${ }^{a}$, C. B. Lang ${ }^{a}$, Markus Limmer ${ }^{a}$, Thilo Maurer ${ }^{b}$, Daniel Mohler ${ }^{a}$ \\ and Andreas Schäfer ${ }^{b}$ \\ ${ }^{a}$ Institut für Physik, FB Theoretische Physik, Universität Graz, A-8010 Graz, Austria \\ ${ }^{b}$ Fakultät für Physik, Universität Regensburg, D-93040 Regensburg, Germany \\ E-mail: christof.gattringereuni-graz.at, christian.langeuni-graz.at, \\ markus.limmerduni-graz.at thilo.maurerdphysik.uni-regensburg.de, \\ daniel.mohler@uni-graz.at \\ andreas.schaeferdphysik.uni-regensburg.de
}

We present recent results of our dynamical simulations with Chirally Improved fermions and report on new developments in the determination of excited light-quark meson states using interpolators constructed by applying covariant derivatives on Jacobi-smeared quark sources within the framework of the variational method.

The XXVI International Symposium on Lattice Field Theory

July 14-19 2008

Williamsburg, Virginia, USA

\footnotetext{
* Speaker.
} 


\section{Introduction}

Simulations with full chiral symmetry using Overlap fermions are still in their infancy. Currently the computational cost of such simulations prohibits large-scale simulations of fermions fulfilling the Ginsparg-Wilson (GW) relation exactly. Therefore, we are working with an approximate algebraic solution to the GW relation [1, 2]. These so-called Chirally Improved (CI) fermions are still computationally demanding as the fermion action includes several hundred terms ranging up to three links distances.

Simulations with CI fermions in the quenched approximation have demonstrated good chiral and scaling behavior [3]. Interpolators constructed from Jacobi-smeared sources [4, 5] of different width as well as interpolators containing derivatives have been used to extract the spectrum of light mesons [6, 7] within this approximation. For the extraction of excited states the variational method [8, 9] has been used, which also enables one to separate contributions from ghost states [10].

Here we present a progress report of ongoing dynamical simulations with CI fermions. The focus will be on the mass spectrum and we will present preliminary results for both mesons and baryons. Preliminary results on the simulation and hadrons masses have previously been presented in [11, 12]. For a discussion of other recent results for excited states see [13].

\section{Details of the simulation}

For our simulations with dynamical quarks we use the Lüscher-Weisz gauge action and two mass degenerate CI fermions. We use a a standard HMC algorithm with mass preconditioning [14] and a mixed-precision inverter. Our action also incorporates one level of stout smearing. Table 1 shows the simulation parameters for our configuration ensembles A-C (lattice size $16^{3} \times 32$ ).

\begin{tabular}{cccccc}
\hline \hline ensemble & $\beta_{\mathrm{LW}}$ & $m_{0}$ & HMC time & $a[\mathrm{fm}]$ & $m_{\pi}[\mathrm{MeV}]$ \\
\hline A & 4.70 & -0.050 & 591 & $0.1507(17)$ & $526(7)$ \\
B & 4.65 & -0.060 & 1108 & $0.1500(11)$ & $469(4)$ \\
C & 4.58 & -0.077 & 1046 & $0.1440(11)$ & $318(5)$ \\
\hline \hline
\end{tabular}

Table 1: Run parameters, lattice spacing and pion masses for ensembles A-C of lattice size $16^{3} \times 32$. The scale has been set using the Sommer parameter $r_{0, \text { exp }}=0.48 \mathrm{fm}$.

The lattice spacing has been determined from the static quark potential (for hypercubic smeared configurations). We fit to the potential

$$
V_{L}(r)=A+\frac{B}{r}+\sigma r+c_{3} \Delta V(r),
$$

where $\Delta V(r) \equiv\left[\frac{1}{\mathbf{r}}\right]-\frac{1}{r}$ contains the perturbative lattice Coulomb potential $\left[\frac{1}{r}\right]$. Using a value of $r_{0, \exp }=0.48 \mathrm{fm}$ for the Sommer parameter we can then determine the lattice spacing as follows:

$$
a=r_{0, \exp } \sqrt{\frac{\sigma}{1.65+B}} .
$$

Figure 1 shows example fits to the logarithm of the Wilson loop expectation value $\ln W(r, t)$ and to the potential for ensemble $\mathrm{C}$. 

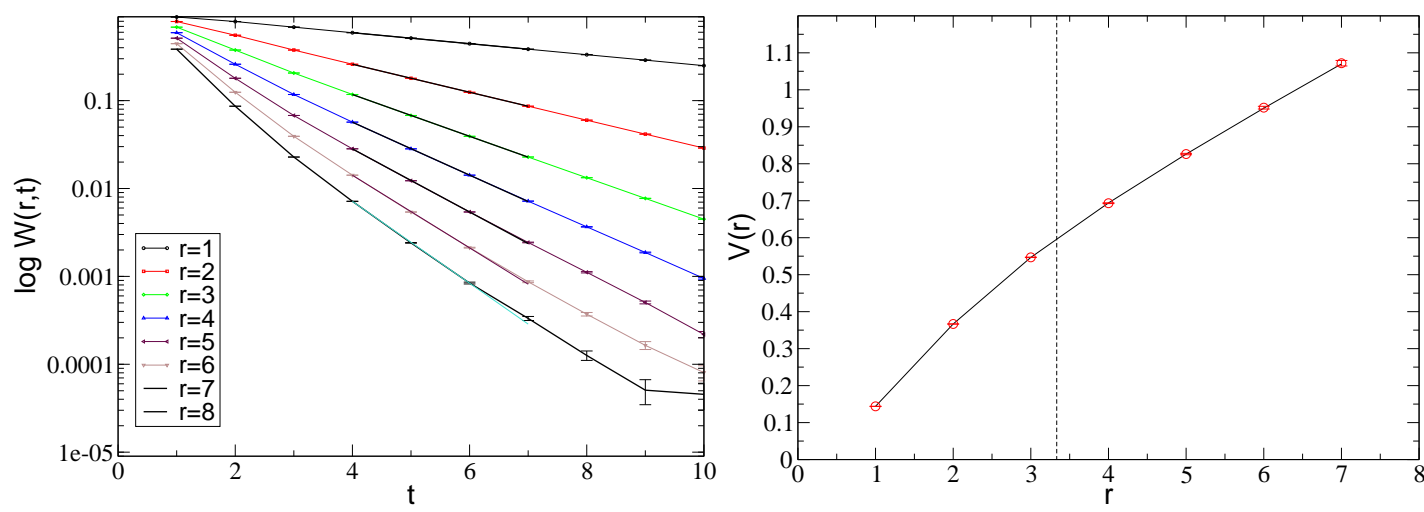

Figure 1: L.h.s.: Fit to $\ln W(r, t)$ in the range $4 \leq t \leq 7$. R.h.s: Fit to the potential in the range $1 \leq r \leq 7$. The data points have been connected by straight lines to guide the eye.

\section{Quark sources for the variational method}

\subsection{Variational method}

For the extraction of hadron masses we use the variational method [8, 9]. One uses a matrix of correlators projected to fixed (here: zero) spatial momentum

$$
C(t)_{i j}=\sum_{n}\left\langle 0\left|O_{i}\right| n\right\rangle\left\langle n\left|O_{j}^{\dagger}\right| 0\right\rangle
$$

and solves the generalized eigenvalue problem

$$
C(t) \vec{v}_{k}=\lambda_{k}(t) C\left(t_{0}\right) \vec{v}_{k}, \quad \lambda_{k}(t) \propto \mathrm{e}^{-t M_{k}}\left(1+\mathscr{O}\left(\mathrm{e}^{-t \Delta M_{k}}\right)\right) .
$$

At sufficiently large time separation each eigenvalue receives contributions from only a single mass. At the same time the eigenvectors can serve as a fingerprint to identify the states when followed over several $t$-values. For a recent discussion of the generalized eigenvalue problem in that context see [15].

\subsection{Quark sources}

We construct interpolators from Jacobi-smeared [4, 5] quark sources $S$ of different width, with the same choice of the parameters $\kappa$ and $N$ as given in [6].

$$
\begin{aligned}
S & =M S_{0} \quad \text { with } \quad M=\sum_{n=0}^{N} \kappa^{n} H^{n} \quad \text { and } \\
H(\vec{n}, \vec{m}) & =\sum_{j=1}^{3}\left(U_{j}(\vec{n}, 0) \delta(\vec{n}+\hat{j}, \vec{m})+U_{j}(\vec{n}-\hat{j}, 0)^{\dagger} \delta(\vec{n}-\hat{j}, \vec{m})\right) .
\end{aligned}
$$

$S_{0}$ denotes a point source. Combinations of such approximately Gaussian sources allow for nodes in the interpolating operators while using fewer quark propagators than in other approaches. In addition to these sources we also use derivative quark sources $W_{d_{i}}$ by applying covariant derivatives to the Gaussian smeared sources:

$$
W_{d_{i}}=D_{i} S, \quad D_{i}(\vec{x}, \vec{y})=U_{i}(\vec{x}, 0) \delta(\vec{x}+\hat{i}, \vec{y})-U_{i}(\vec{x}-\hat{i}, 0)^{\dagger} \delta(\vec{x}-\hat{i}, \vec{y}) .
$$



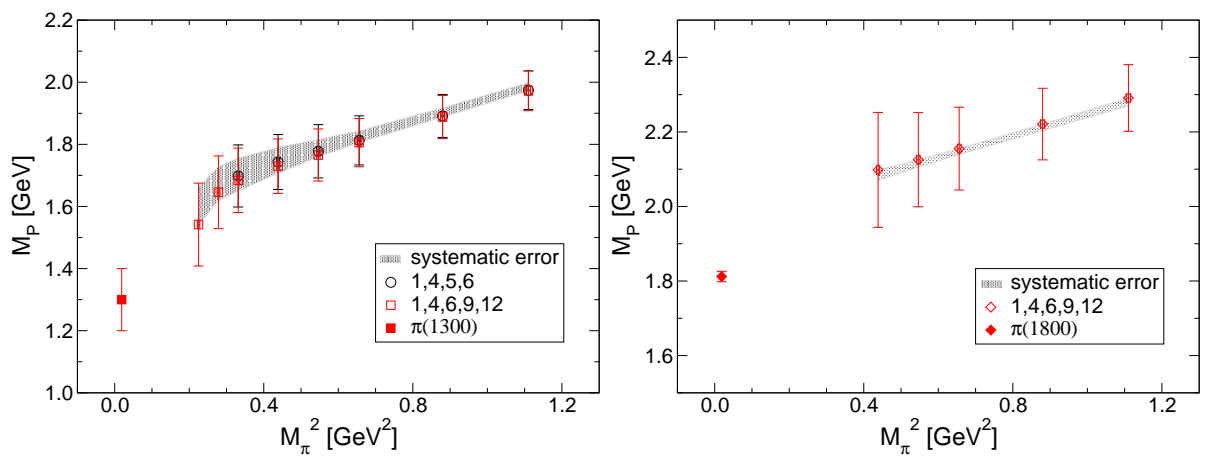

Figure 2: 1 st and 2nd excitation of $\pi$. The shaded region indicates the systematic uncertainty due to the choices of fit-range and interpolator combination.

\section{Spectrum results}

In this section we present results from quenched lattices as well as preliminary results from the simulation with dynamical CI quarks.

\subsection{Results from quenched lattices}

Figure 1 shows results for the pion channel based on 99 quenched configurations. A clear signal can be obtained for both the first and the second excitation. The results from the set of interpolators containing both Gaussian and derivative sources enable us to identify the second excited state which (on the same dataset) cannot be observed with the Gaussian sources alone. For more details, some further plots and results from other channels see [7].

\subsection{Results from dynamical lattices}

For the dynamical CI lattices, the lattice size is $16^{3} \times 32$, corresponding to a spatial extent of $(2.4 \mathrm{fm})^{3} \times 4.8 \mathrm{fm}$. We analyze every fifth configuration and shift the source positions for consecutive configurations to reduce autocorrelation. All data presented are of a preliminary nature. Table 2 provides an overview of the current ensembles.

\subsubsection{Results for baryons}

Figure 3 A shows results for the mass of the (positive parity) nucleon ground state. The empty symbols indicate a nucleon made from valence quarks that are heavier than the sea quarks while the

\begin{tabular}{lccccc}
\hline ensemble & \# conf.s & $a[\mathrm{fm}]$ & $m_{\mathrm{AWI}}[\mathrm{MeV}]$ & $m_{\pi}[\mathrm{MeV}]$ & $a m_{\pi} L$ \\
\hline A & $100 / 100$ & $0.1507(17)$ & $43.0(4)$ & $526(7)$ & 6.4 \\
B & $100 / 200$ & $0.1500(11)$ & $35.1(2)$ & $469(4)$ & 5.8 \\
C & $100 / 200$ & $0.1440(11)$ & $15.0(4)$ & $318(5)$ & 3.8 \\
\hline
\end{tabular}

Table 2: Dynamical CI runs. \# conf.s refers to the number of (independent) configurations analyzed for mesons or baryons respectively. Values in physical units have been obtained as described in Section 2. 

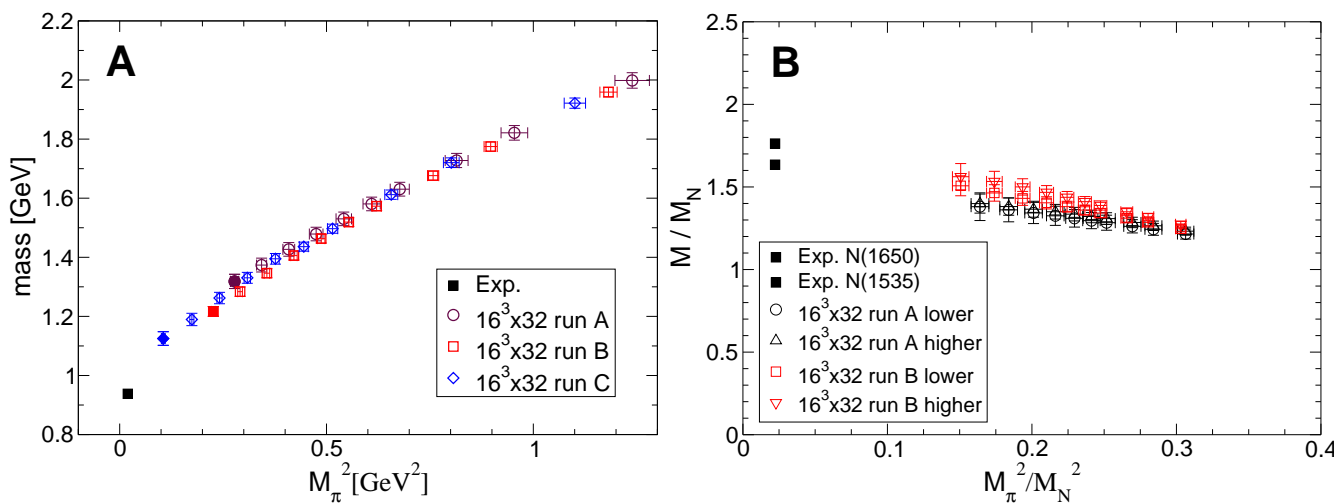

Figure 3: A: Mass of the (positive parity) nucleon ground state; B: Mass of the negative parity ground state and 1 st excited state.
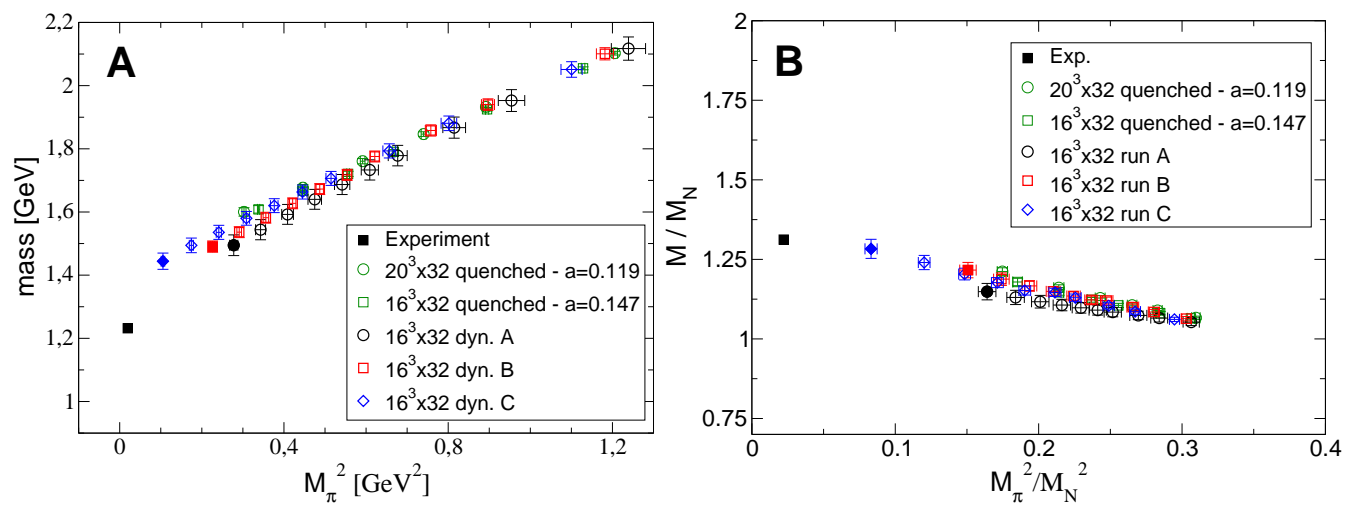

Figure 4: A: Ground state in the Delta channel in physical units; B: Same plotted in units of the nucleon mass.

full symbols indicate the fully dynamical, unitary point for the respective runs. The error bars are purely statistical, also including the statistical error in the determination of the scale. Systematic errors from varying fit ranges and combinations of interpolators are not included in the error bars. Since we have set the scale individually for each of the parameter sets this amounts to a mass dependent renormalization scheme. We therefore cannot use ChPT extrapolations valid in the mass independent scheme. More runs at different masses and volumes will be necessary for reliable extrapolations to the physical points.

Figure $3 \mathrm{~B}$ shows the ground and first excited state masses observed in the negative parity channel. While the statistical errors for the different runs show a deviation a more careful analysis of the systematics due to the choice of interpolators and fit ranges will be necessary. The quality of the data for run $\mathrm{C}$ was not sufficient to fit these states at the unitary point, therefore it has been omitted from the plot.

Figure 4 shows the ground state mass in the $\Delta$-channel plotted in physical units and in units of the nucleon mass. The $\Delta^{++}$is a resonance, which complicates the interpretation of the ground state signal. To get a clear interpretation an analysis of this state in different volumes will have to be 
performed. We observe that when plotting the $\Delta^{++}$in units of the nucleon mass the finite volume effects at small pion masses cancel, thus leading to consistent data for all three runs.
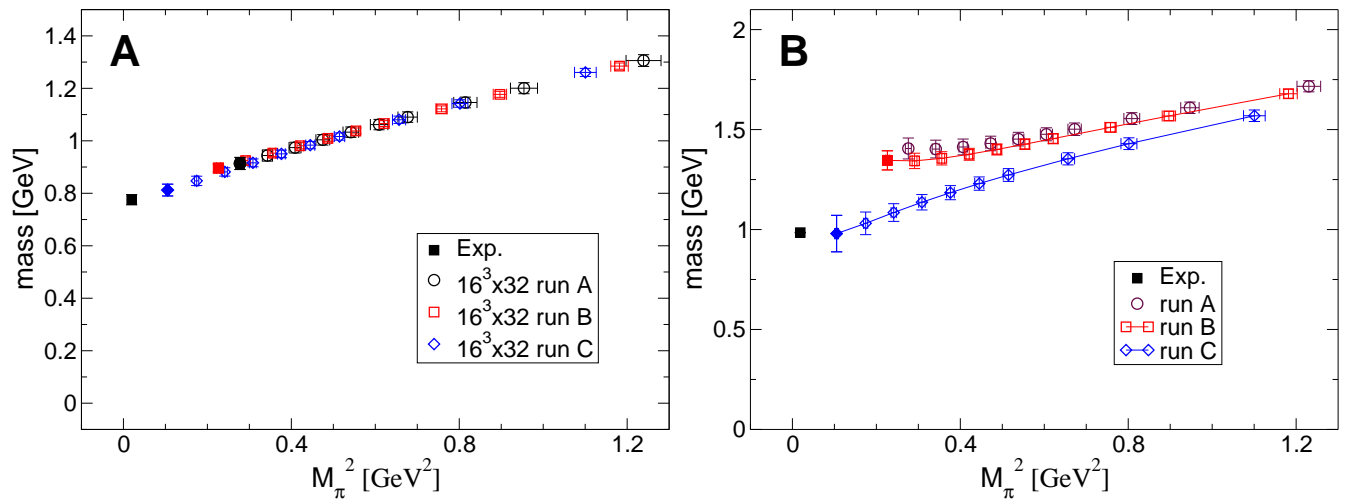

Figure 5: A: Ground state in the $1^{--}$channel ( $\rho$-meson); B: Ground state signal in the $0^{++}$channel for interpolators 9,10 in the notation of [7].

\subsubsection{Results for mesons}

For the mesons the set of interpolators containing derivative sources described in detail in [7] has been used. As can be seen in Table 2, only a subset of configurations was used for this preliminary analysis. Figure 5 A shows some results for the ground state of the $\rho$ meson. Figure $5 \mathrm{~B}$ displays results for the lowest energy level in the isovector scalar $a_{0}$ channel for one possible set of interpolators. We point out, however, that our data show a large operator dependent variation. Most quenched results ${ }^{1}$ indicate that the ground state in this channel might be the $a_{0}(1450)$, in agreement with a tetraquark interpretation of the $a_{0}(980)$. Results from dynamical simulations lead to somewhat lower states, possibly due to the presence of $\pi \eta$ scattering states. A systematic analysis will have to take into account several volumes to discriminate scattering states from bound states. One possible interpretation of our results would be a crossing of the lowest energy level at the quark mass where the $\pi \eta$ state becomes lower than the $a_{0}(980)$.

\section{Conclusions}

We presented a progress report on spectroscopy with dynamical CI fermions. We demonstrated that good signals can be obtained for (most) ground state mesons and baryons. Emphasis has been put on describing the methods and we are currently refining these by experimenting with different gauge link smearings. It would be desirable to perform further runs with the CI action to control both discretization and finite volume effects and to be able to use a mass independent renormalization scheme.

\footnotetext{
${ }^{1}$ Notice that the analysis of the quenched data is made difficult by artifacts, so called "ghosts".
} 


\section{Acknowledgments}

The calculations have been performed on the SGI Altix 4700 of the Leibniz-Rechenzentrum Munich and on local clusters at ZID at the University of Graz. We thank these institutions for providing support. M.L. and D.M. are supported by "Fonds zur Förderung wissenschaflicher Forschung in Österreich" (DK W1203-N08). A.S. and T.M. acknowledge support by DFG and BMBF. The work has been supported by DFG project SFB TR55.

\section{References}

[1] C. Gattringer, A new approach to Ginsparg-Wilson fermions, Phys. Rev. D63 (2001) 114501, [hep-lat/0003005].

[2] C. Gattringer, I. Hip, and C. B. Lang, Approximate Ginsparg-Wilson fermions: A first test, Nucl. Phys. B597 (2001) 451-474, [hep-lat/0007042].

[3] BGR Collaboration, C. Gattringer et al., Quenched spectroscopy with fixed-point and chirally improved fermions, Nucl. Phys. B677 (2004) 3-51, hep-lat/0307013.

[4] S. Güsken et al., Nonsinglet axial vector couplings of the baryon octet in lattice QCD, Phys. Lett. B227 (1989) 266.

[5] C. Best et al., Pion and rho structure functions from lattice QCD, Phys. Rev. D56 (1997) 2743-2754, [hep-lat/9703014].

[6] T. Burch et al., Excited hadrons on the lattice: Mesons, Phys. Rev. D73 (2006) 094505, [hep-lat/0601026].

[7] C. Gattringer, L. Y. Glozman, C. B. Lang, D. Mohler, and S. Prelovsek, Derivative sources in lattice spectroscopy of excited mesons, Phys. Rev. D78 (2008) 034501, [arXiv:0802.2020].

[8] C. Michael, Adjoint sources in lattice gauge theory, Nucl. Phys. B259 (1985) 58.

[9] M. Lüscher and U. Wolff, How to calculate the elastic scattering matrix in 2-dimensional quantum field theories by numerical simulation, Nucl. Phys. B339 (1990) 222.

[10] T. Burch, C. Gattringer, L. Y. Glozman, C. Hagen, and C. B. Lang, Variational method for lattice spectroscopy with ghosts, Phys. Rev. D73 (2006) 017502, [hep-lat/0511054].

[11] C. B. Lang, P. Majumdar, and W. Ortner, QCD with two dynamical flavors of chirally improved quarks, Phys. Rev. D73 (2006) 034507, [hep-lat/0512014].

[12] R. Frigori et al., Dynamical Chirally Improved quarks: First results for hadron masses, PoS LAT2007 (2007) 114, [arXiv:0709.4582].

[13] C. Morningstar, plenary talk at LATTICE 2008, to be published in PoS(LAT2008)

[14] M. Hasenbusch, Speeding up the Hybrid-Monte-Carlo algorithm for dynamical fermions, Phys. Lett. B 519 (2001) 177, hep-lat/0107019].

[15] B. Blossier, G. von Hippel, T. Mendes, R. Sommer, and M. Della Morte, Efficient use of the generalized eigenvalue problem, arXiv:0808.1017. 